\title{
LE HÉROS VIRTUEL COMME RÉVÉLATEUR DE FANTASMES ARCHAÏQUES À L'ADOLESCENCE
}

\author{
Xanthie Vlachopoulou et al.
}

ERES | Dialogue

$2014 / 1-n^{\circ} 203$

pages 111 à 120

ISSN 0242-8962

Article disponible en ligne à l'adresse:

http://www.cairn.info/revue-dialogue-2014-1-page-111.htm

Pour citer cet article :

Vlachopoulou Xanthie et al., « Le héros virtuel comme révélateur de fantasmes archaïques à l'adolescence », Dialogue, 2014/1 n²03, p. 111-120.

Distribution électronique Cairn.info pour ERES.

(c) ERES. Tous droits réservés pour tous pays.

La reproduction ou représentation de cet article, notamment par photocopie, n'est autorisée que dans les limites des conditions générales d'utilisation du site ou, le cas échéant, des conditions générales de la licence souscrite par votre établissement. Toute autre reproduction ou représentation, en tout ou partie, sous quelque forme et de quelque manière que ce soit, est interdite sauf accord préalable et écrit de l'éditeur, en dehors des cas prévus par la législation en vigueur en France. II est précisé que son stockage dans une base de données est également interdit. 


\title{
Le héros virtuel comme révélateur de fantasmes archaïques à l'adolescence
}

\author{
Xanthie Vlachopoulou, Lise Haddouk, \\ Florian Houssier, Sylvain Missonnier
}

Mots-clés

Adolescence, fantasme, héros, virtuel.

\section{Résumé}

La figure du héros ne se limite plus à celle du héros mythique ; le héros virtuel en constitue désormais une transformation contemporaine pour occuper cette fonction dans la vie fantasmatique des adolescents. Mais ces deux héros recouvrent-ils les mêmes enjeux internes ? À travers l'analyse d'un cas lié à une recherche clinique, l'article propose d'explorer l'investissement de l'avatar héroïque au centre du processus d'adolescence, au moyen de trois axes : I'effervescence pulsionnelle, la conflictualité narcissico-objectale et la problématique de perte. Ce qui ressort de la comparaison par analogie entre l'investissement libidinal des héros antique et virtuel relève de l'écart qui s'instaure dans cette situation clinique entre imaginer être un héros et alimenter virtuellement le fantasme mégalomaniaque d'être le héros.

«Autrefois, ils pensaient devenir des héros: ils sont devenus des jouisseurs. Le héros leur fait horreur et les effraie."

F. W. Nietzsche, Ainsi parlait Zarathoustra, 1883-1885

\footnotetext{
Xanthie Vlachopoulou, psychologue clinicienne, docteur en psychologie clinique et psychopathologie, membre associé du laboratoire LPCP, EA4056, Paris Sorbonne Cité; xanthievlachopoulou@yahoo.fr Lise Haddouk, psychologue clinicienne, docteur en psychologie clinique et psychopathologie ;

lise.haddouk@gmail.com

Florian Houssier, psychologue, psychanalyste, professeur de psychologie clinique et de psychopathologie, Unité transversale de recherches : psychogenèse et psychopathologie (UTRPP), Université Paris-Nord, Villetaneuse, Paris Sorbonne Cité ; houssier.florian@gmail.com

Sylvain Missonnier, psychologue, psychanalyste, professeur de psychopathologie clinique de la périnatalité et de la première enfance, université Paris-Descartes, laboratoire LPCP, EA 4056 ; syl@carnetpsy.com
} 


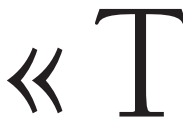

out le monde pensait que j'étais extraordinairement puissant. [...] Un jour, il y a le dieu des dieux qui est venu pour enterrer le roi et il avait convié les prêtres de toutes les religions à venir. Donc, je me retrouve avec le dieu des dieux et les autres prêtres [...] je commence à faire les poches des autres grands prêtres, puis à un moment je me dis, si j'essayais de faire les poches du dieu des dieux. Et là, je vois un milliard de pièces d'or. Et je me dis, obligé, je lui vole. Donc, je lui pique tout son sac. Conséquence : ils ont fait mon procès public. Sur une place publique, j'ai été jugé coupable de vol, j'ai été maudit. » Les propos de ce patient imprégnés d'une essence mythique intriguent par la coexistence de la modernité de l'adolescence et de leur proximité avec les récits héroïques «classiques». Son contenu ne permet pas de situer ce récit dans le temps; seule la forme discursive trahit sa modernité. Les jeux vidéo ouvrent sur la possibilité d'incarner un héros pour vivre des aventures extraordinaires. Dans sa description des jeux en ligne, Cario (2010) souligne d'emblée l'opposition des deux mondes : celui dans lequel nous évoluons en chair et en os et celui qui laisse place aux pixels: "Chaque jour, ces voyageurs du virtuel quittent momentanément la réalité pour retrouver sur internet des univers où l'environnement est tantôt médiéval, tantôt futuriste, où les normes sociales se trouvent bouleversées et où ils peuvent vivre, en compagnie d'amis qu'ils n'ont jamais croisés physiquement, des aventures mémorables.»

Valleur (2002) parle à propos des jeux MMORPG ${ }^{1} \mathrm{~d}^{\prime}$ « engloutissement passionnel », remarquant même au sujet des adolescents ou de jeunes adultes, joueurs excessifs, que par cette pratique « ils viennent poser leurs difficultés souvent liées à une période de changement, une transition, quelquefois délicate, s'inscrivant le plus souvent dans une problématique adolescente ${ }^{2} »$. En effet, la nécessité de faire appel à des figures héroïques, de s'identifier à elles et vouloir les incarner traverse les siècles et les civilisations, accompagnant le passage de l'infantile vers le pubertaire (Gutton, 1991). Les héros dans leur jeunesse ont affronté la loi du père pour pouvoir s'affranchir et actualiser leur destin héroïque, comme le montre Rank (1909) dans son analyse de la jeunesse de différents héros tels que Moïse, Jésus, Edipe. Car, tout comme pour les adolescents, ce chemin vers le devenir sujet relève d'un trajet dont l'issue n'est pas réductible à une simple question de temps matériel, mais bien d'enjeux conflictuels inconscients inhérents à la réalité psychique. "Dans le pubertaire, l'enfant suit tragiquement le destin d'Edipe. Par l'adolescens, il désexualise la violence de ses pulsions et procède à un travail de subjectivation et d'historicité »

1. Ces initiales correspondent à l'appellation anglophone : "Massively Multiplayers On Line Role Playing Games ", traduite en français par l'expression «Jeux de rôle en ligne massivement multijoueurs ».

2. Rapport d'activité 2004, Hôpital Marmottan : http ://www.hopital-marmottan.fr 
(Gutton, 1991, p. 12). C'est ainsi que le devenir adulte s'annonce. Cela peut expliquer l'intérêt des adolescents pour les héros, qui prennent des formes diverses à travers les époques et les cultures, courant des héros mythiques à des formes plus actuelles de figures héroïques, comme les héros de jeux vidéo. Mais est-ce que ces héros pixélisés occupent une fonction similaire à celle des héros mythiques auprès des adolescents?

Par l'analyse d'une situation clinique, nous explorons les maniements possibles de ces héros numériques par les adolescents qui figurent à travers leurs écrans des personnages pouvant prendre une place centrale dans leur vie fantasmatique. Nous analyserons la fonction du héros virtuel en la dialectisant avec celle du héros mythique, toutes deux articulées à trois axes majeurs au cour du processus adolescent qui guideront notre réflexion: effervescence pulsionnelle, conflictualité narcissico-objectale et problématique de perte.

\section{De l'investissement fantasmatique de la figure du héros}

Chaque mythe est enrichi par la vérité polyphonique qu'il nous transmet. Par analogie, avec certains patients il est parfois indispensable de reprendre sous des formes différentes le même contenu pour que le sujet puisse s'en approprier un des sens latents. En tant que concentré de figurations symboliques, le mythe organise une représentation en récit de l'irreprésentable, ou du refoulé originaire. Les fantasmes originaires y trouvent de nouvelles liaisons qui permettent une symbolisation de second degré par la figurabilité (Tasca, Seabrea, 2002). La principale caractéristique du mythe est d'être l'objet d'une reconstruction, comme en témoigne la pluralité des versions de chaque mythe. Par ses manques ou ses énigmes, il pousse à l'interrogation et à l'inférence (Moscovici, 1993). Le héros mythique est une figure qui se décline sous des formes très variées dans la culture : héros de bandes dessinées, contes, légendes, avatars et héros de jeux vidéo. Le héros mythique implique l'identification à un personnage central qui mobilise l'investissement narcissique - projection d'une part de soi - et objectal identification à une représentation symbolique insérée dans un récit.

Revenons sur la question du héros antique tel qu'il apparaît dans la littérature psychanalytique. La référence au héros renvoie à la question de l'inscription du sujet dans sa filiation, qui marque l'interdit de l'inceste et la différence des générations. Rank (1909) interprète ces légendes dans le sens d'un conflit avec le père, par la révolte qui transforme le héros en réformateur. Pourtant, il ne suit pas seulement la piste odipienne tracée par Freud ; avec l'analyse des origines des héros, il confronte ces données mythiques aux fantasmes prégénitaux de l'enfant et en vient à concevoir 
le mythe comme l'expression de fantasmes régressifs par les adultes. Rank (1909, p. 101-102) explique ainsi : "Lapplication de ces théories à notre schéma fait apparaître une concordance entre la tendance du roman familial et celle du mythe du héros, ce qui nous autorise à établir une analogie entre le moi de l'enfant et le héros de la légende. Rappelons que le mythe exprime en général une tendance de séparation d'avec les parents et que le même désir se réveille dans les fantasmes du jeune individu au moment où il cherche à obtenir son indépendance et son autonomie. Le moi de l'enfant se comporte en cela comme le héros de la légende. [...] Le fantasme du roman familial apparaît donc simplement réalisé dans le mythe à travers un audacieux renversement des situations réelles. »

La même année, Freud (1909, p. 158) fait émerger la notion de roman familial : "Une activité fantasmatique [...] qui se manifeste tout d'abord dans les jeux de l'enfance pour s'emparer ensuite, à partir environ de l'époque pubertaire, du thème des relations familiales. Un exemple caractéristique de cette particulière activité fantasmatique est ce que l'on connaît bien sous le nom de rêve diurne. »Cette activité fantasmatique prend pour tâche de se débarrasser des parents, désormais dédaignés, et de leur en substituer d'autres, en général d'un rang social plus élevé. La rêverie diurne joue un rôle important au moment de l'adolescence ; elle représente un agent de transformation qui assure le passage de l'infantile vers le pubertaire. À l'adolescence, les jeux vidéo s'inscrivent-ils dans la continuité de cette modalité de l'activité fantasmatique (Houssier, Marty, 2010) ou, a contrario, sont-ils une entrave au processus dans le sens d'une activité antireprésentative ? Chaque usager adolescent apporte une réponse unique et évolutive à cette interrogation. Dans cette perspective clinique résolument casuistique, la situation clinique que nous présentons articule, sur fond de dépendance à « l'objet virtuel » (Missonnier, 2007), des indices à la compréhension de cette question.

\section{Pierre, entre angoisse d'abandon et création du double}

Pierre est un jeune homme de 19 ans que nous avons rencontré dans le cadre de notre recherche ${ }^{3}$ sur le recours excessif aux jeux vidéo en ligne durant le processus adolescent. Cadet d'une fratrie de quatre enfants, Pierre vit chez ses parents et est actuellement en début d'études dans le domaine des télécommunications. Il se présente comme un jeune homme surinvestissant l'usage de jeux de rôles en ligne, et ce, depuis plusieurs années.

3. X. Vlachopoulou, «Le virtuel et ses destins: quand la virtualité adolescente rencontre le virtuel des nouvelles technologies de l'information et de la communication », thèse de doctorat soutenue le 7 octobre 2011, université Paris-Descartes, effectuée sous la direction du Pr F. Marty. 


\section{Le héros virtuel au service de l'ascétisme}

De la rencontre avec Pierre est ressortie sa difficulté à faire face à la réactivation pulsionnelle postpubère. Manifestant une grande inhibition et une tendance à la restriction, mais aussi une pensée peu créative et un effort pour maintenir le conflit à distance, il se défend contre des émergences pulsionnelles intenses, qu'elles soient libidinales ou agressives, sous-tendues par des fantasmes œdipiens qui affleurent avec peu de refoulement et viennent interpeller le clinicien sur leur consistance et leur éventuel enracinement archaïque.

En parlant du jeu, la tonalité de son discours évolue, il semble s'animer. La place du jeu vidéo dans sa vie parâit très importante, il dit même, à un moment : "Moi, c'est City Of Heroes [nom du jeu]. » Une dimension de plaisir apparaît, notamment par la sensation de maîtrise que lui procure cette pratique, comme il le signifie avec une expression particulièrement révélatrice : " J'ai choisi cet univers car il est facile à prendre en main. »Cet aspect de maîtrise est très bien illustré par le choix de son personnage, qui est un « contrôleur », permettant de maîtriser, dans l'emprise, de loin, la relation aux autres joueurs, sans implication corporelle, même par avatars interposés. Son choix d'archétype ${ }^{4}$ au niveau des batailles virtuelles est assez intéressant à analyser, car, comme il l'évoque, c'est un type de personnage « qui contrôle le pouvoir, enfin les sorts qu'ils ont, c'est beaucoup de sorts où ils immobilisent les pers... les mob ${ }^{5}$, donc, ils ne ne peuvent plus bouger, soit ils sont entravés, soit ils ne peuvent plus se défendre tout court ».

Sa façon d'attaquer dans le jeu témoigne d'une part de la massivité des fantasmes de destruction, avec l'idée de rendre l'adversaire impuissant pour mieux l'achever, et d'autre part de sa tendance au repli par la mise à distance des autres. Il est intéressant de souligner à ce propos son lapsus, lorsqu'il commence par dire qu'il immobilise des personnes ou personnages dans ce mot qu'il ne termine pas ("pers...») et qu'il reprend de suite en le remplaçant par "mob», qui désigne des avatars automatisés. Ceux-ci ne sont donc pas gérés par d'autres joueurs mais par des programmes: les attaques ne peuvent pas être mises en scène envers d'autres personnes, même par avatars interposés, son choix d'adversaire se portant sur des avatars robotisés.

4. Modèle primitif, idéal : ce terme est utilisé en 1850 pour désigner ce qui est « original ou exemple caractéristique servant de modèle à un genre de choses ». Il est utilisé dans le langage des jeux vidéo pour désigner un personnage-type qui correspond à un concept facilement identifiable.

5. Terme utilisé par les joueurs pour désigner un groupe d'ennemis gérés par l'ordinateur. 
Ainsi, tout en bénéficiant a minima du virtuel des MMORPG, dans leur dimension non charnelle, il semblerait que la tendance à la répression du mouvement pulsionnel persiste, y compris dans ces espaces virtuels dans lesquels il évite les confrontations. À la différence des récits fictifs relatifs aux héros mythiques, le fait, pour Pierre, d' « incarner » un héros virtuel n'offrirait pas la distance nécessaire au déploiement des fantasmes dans le sens où l'adolescent tendrait à se répandre en fantasmes (Freud, 1905).

\section{Dans la peau de l'avatar}

Concernant l'axe narcissico-objectal, il nous est apparu que Pierre présente des assises narcissiques fragiles entravant le passage vers des relations objectales non incestueuses. À notre invitation de se présenter, il répond : « D'accord, donc Pierre mon prénom, j'ai 19 ans, je fais des études dans les télécom. Je suis célibataire, j'habite encore chez mes parents. J'aime bien les jeux vidéo, le sport, même si je ne pratique pas beaucoup. » Sa présentation nous laisse l'impression que Pierre répond à un questionnaire en remplissant mentalement des cases - «Pierre mon prénom ». Il décrit aussi un environnement familial qui ne semble pas très attentif à lui. Lattention des parents ne serait pas à la hauteur de son souhait. Une déception semble apparaître quand il évoque le fait qu'ils n'ont pas vraiment pris conscience que sa pratique est excessive : "Ils n'ont pas vu la différence avec les jeux auxquels je jouais avant. »

Dressant le portrait d'une vie solitaire avec un choix d'études peu stimulant et un environnement familial qui ne le comprend pas, Pierre donne l'impression de se trouver dans l'impasse d'une vie décourageante et ennuyeuse, signe d'une dépressivité assez manifeste. Sa créativité semble n'avoir jamais été valorisée par les autres et lui-même tente de la réprimer. Mais le jeu, bienveillant, donne des récompenses. De plus, dans ce jeu, il n'a pas à se confronter à la rivalité avec ses pairs: "Quand il y a une récompense, tout le monde l'a, bon c'est pas forcément celle que tout le monde veut, mais tout le monde en a une. »

Malgré un discours assez défensif autour de la question d'incarner un super héros - «C'est sympa, mais bon, on s'identifie pas trop à son personnage... moi je sais que c'est pas du tout le cas »-, il arrive à reconnaître le plaisir de cette incarnation héroïque : "Oui c'est sympa de jouer un super héros, mais voilà. » Toujours dans un mouvement de banalisation de ce choix d'avatar, il nous dit que le prénom choisi pour son personnage est « plus ou moins [celui du] dieu de la création [...] et je crois aussi que c'est le père de toutes les autres divinités », rajoutant défensivement que « c'est juste parce que le nom m'avait plu». 
Là encore, la fonction du héros virtuel ne correspond pas à celle du héros mythique. Si, dans la mythologie, le sujet s'identifie au héros, dans les jeux vidéo le moi est projeté dans le double que représente l'avatar. Dans le premier cas, on se situerait alors plutôt du côté de l'idéal du moi, dans le second, davantage du côté du moi idéal. Pour rappel, le moi idéal concerne l'identification primitive à un objet encore peu différencié ; l'enfant désire alors être l'autre, par assimilation et introjection. Lidéal du moi relève d'une identification secondaire, souvent au parent qui représente l'interdit œdipien ; l'enfant désire avoir ce que le parent a, dans le sens de la promesse oedipienne qu'il lui lance : «Plus tard, tu pourras... »-sousentendu : " avoir ce que j'ai ». Le héros virtuel produit ainsi une satisfaction plus directe, mais au prix d'être moins constructive ; l'avatar implique une renarcissisation éphémère et ainsi toujours à reprendre au détriment de l'investissement corporel du joueur.

Pierre paraît aussi chercher un soutien narcissique dans les mondes virtuels. Des angoisses d'abandon majeures apparaissent : il semble très réticent à aller à la rencontre de l'autre, même avec la carapace soutenante de son avatar. Se décrivant comme « solitaire », il dit pourtant préférer « les jeux où on joue avec quelqu'un parce que tout seul j'ai un peu de mal... ça fait des années que je ne joue plus tout seul, ça ne m’intéresse plus ». Cette socialisation virtuelle que le jeu lui permet semble être importante pour lui : "Les gens se connaissent et il y a eu quelques IRL ${ }^{6}$, ce qui fait qu'au final on côtoie des personnes et on apprécie d'autant plus, c'est sympa. » Mais, même dans le jeu, la crainte de se trouver seul ou d'être abandonné se dessine encore : "J'ai rarement joué souvent avec les mêmes personnes, parce qu'au bout d'un moment il y a quelqu'un qui me laissait, donc je changeais. »Pierre, avec la crainte d'investir les autres joueurs dans leur singularité au sein d'une relation privilégiée, potentiellement porteuse d'abandon, utilise cet espace pour ne pas se sentir seul.

À contre-courant du héros mythique « seul contre tous » et « réformateur », le héros virtuel évolue dans un monde " massivement multijoueurs », n'est donc jamais seul et ne s'inscrit nullement en rébellion contre les règles. Cependant, concernant la question de la relation d'objet, ces deux héros semblent, tout en optant pour des chemins très différents, offrir une illusion de complétude ; le héros mythique en érotisant la solitude et le héros virtuel en se fondant dans la masse. En effet, les propos de Pierre ne sont pas sans rappeler le titre de l'ouvrage de Turkle (2012) Alone together ${ }^{7}$ évoquant un espace où les rencontres restent virtuelles.

6. IRL : In Real Life (dans la vraie vie).

7. "Alone together " peut être traduit par « seuls ensemble». 


\section{Rester « connecté » à tout prix}

Pierre semble mis à mal dans sa tentative d'élaboration de la perte et nous percevons une difficulté notable dans la possibilité de se représenter l'absence ; ainsi, il confère une part d'irréversible à la séparation vécue alors comme un abandon douloureux. Dans son recours à ces univers persistants, il évite le vécu de solitude et maintient l'illusion d'être « connecté » aux autres, colmatant ainsi ses angoisses d'abandon. En effet, lorsque nous lui avons demandé ce qu'il ferait si quelqu'un lui proposait d'échanger ou d'acheter son personnage, il a répondu : "C'est pas des personnages que j'ai envie d'effacer [...], ce serait bête d'effacer des personnages sur lesquels on a passé beaucoup de temps. » Dans son récit, le fait de se séparer de son avatar est, d'emblée, l'équivalent d'une disparition, d'un effacement de ce dernier. Cette crainte de la perte apparaît même dans son usage de la télévision. Il nous parle en effet d'un programme qu'il « essaye de voir régulièrement » et poursuit en expliquant qu'« il y a tellement de séries que c'est difficile de tout suivre, j'ai pas envie d'avoir de problèmes si je les loupe ». La perte et la séparation semblent ainsi inenvisageables. C'est dans cette même difficulté que s'inscrit sa réponse concernant l'idée d'un arrêt possible du jeu : "Après, il faudrait trouver le jeu qui puisse me plaire. » Larrêt n'étant pas concevable, il faut tout de suite pouvoir trouver un objet de remplacement, pour faire ainsi l'économie de la souffrance d'une absence de l'objet.

Dans les mondes virtuels, Pierre trouve une réponse face à cette impossibilité d'imaginer et de se représenter l'absence. En restant « connecté », il n'est jamais seul et trouve un refuge dans le virtuel-potentiel des MMORPG. Il donne cette même impression dans son usage de la télévision : " Je la mets en fond [...] je l'allume comme ça, pas spécialement pour la regarder. » Ce défaut de recours à ses objets internes se traduit aussi par la difficulté à mobiliser son imagination ; sa réponse en témoigne lorsque nous lui proposons d'imaginer un avatar sans se soucier des contraintes imposées par le développeur du jeu : "Il y aurait un personnage que j'aurais bien aimé faire, mais on peut pas parce que ça n'existe pas. »

Ainsi, concernant l'élaboration de la perte, nos deux héros vont à nouveau emprunter des chemins différents. Le héros mythique archétypal tend vers l'objet total œedipien via l'identification, l'élaboration de la perte, et le héros virtuel de Pierre vers une relation d'objet partiel ou, mieux, une relation d'objet virtuel(le) via l'introjection et l'incorporation, ici formes préœedipiennes de l'identification proches de l'investissement narcissique du héros virtuel. Pierre, avec son avatar, fige dans un éternel présent le passage adolescent par la persistance de l'investissement narcissique du héros virtuel qui comble l'absence de l'objet. À partir de cette observation 
clinique, nous émettions l'hypothèse que, chez Pierre, ce choix de héros virtuel reviendrait plus à montrer le besoin d'un appui pour fantasmer, une sorte de fantasme assisté par ordinateur, que seul le virtuel peut offrir.

\section{Être un héros ou l'imaginer?}

Si l'analogie entre les héros, antique et virtuel, reste envisageable à travers l'analyse du cas de Pierre, il est un écart qui s'instaure entre imaginer être un héros et alimenter le fantasme mégalomaniaque d'être le héros. La référence au héros nous renvoie également à la question de l'inscription du sujet dans sa filiation, qui marque l'interdit de l'inceste et la différence des générations. Rappelons que, selon Kaës (Nicolle, Kaës, 1985), la filiation implique au moins trois générations et références communes à un mythe des origines.

La jeunesse du héros mythique met en scène une transgression de la loi paternelle. Le jeune héros, pour pouvoir relever le défi d'être réformateur, se doit d'être transgressif, il entre en conflit avec l'ordre symbolique de l'ancêtre fondateur et en met en exergue la malléabilité, gage de pérennité. Lidentification au héros virtuel est un temps parfois nécessaire pour l'adolescent, mais, comme dans le cas précis de Pierre, poussé à l'extrême d'une jouissance régressive, elle peut aller jusqu'à une menace de paralysie du dynamisme réformateur de l'ordre symbolique, jusqu'au sacrifice du sujet en devenir, et constituer une impasse dans son travail de subjectivation. Les fantasmes de rivalité liés à la destruction virtuelle de l'autre, ce représentant de la castration, apparaissent ici comme la surface pseudo-œedipienne des conflits de Pierre ; les fantasmes de destruction, dans le sens de la pulsion de destructivité et de mort, témoignent davantage des aspects régressifs archaïques mis en jeu via son avatar. Cet agissement virtuel destructeur est un équivalent de réponse à des fantasmes persécutifs, engageant dans le même temps une fuite de l'autre incarné, en chair. La rencontre avec l'autre mobilise des fantasmes violents phobogènes, les avatars étant alors un espace au service de fantasmes antipensée (Racamier, 1995).

Une vision psychothérapeutique optimiste (avec un bon cadre) considérera les réactualisations de ces formes préœedipiennes de l'identification comme un tremplin dynamique vers l'œedipification. 


\section{Bibliographie}

Cario, E. 2010. « Jeux en ligne », Encyclopédie Universalis (Version numérique).

FreUd, S. 1905. Trois essais sur la théorie sexuelle, Paris, Gallimard, 2003.

FREUd, S. 1909. « Le roman familial des névrosés ", dans Névrose, psychose et perversion, Paris, Puf, 2010.

HousSIER, F. ; MARTY, F. 2010. " Adolescence, image et rêverie. Destins de la dépendance à l'objet », Cahiers de psychologie clinique, 35, Bruxelles, De Boeck, 77-91.

Gutton, P. 1991. Le pubertaire, Paris, Puf.

Missonnier, S. 2007. "Une relation d'objet virtuelle? ?, Le carnet PSY, 120, Paris, Cazaubon, 43-47.

MoscovicI, M. 1993. « Les préhistoires : pour aborder "Totem et tabou" ", Revue française de psychanalyse, 57, 3, Paris, Puf, 691-712.

Nicolle, O. ; Kä́s, R. 1985. (sous la direction de). L'institution en héritage : mythes de fondation, transmissions, Paris, Dunod, 2008.

Racamier, P. 1995. L'inceste et l'incestuel, Paris, Dunod, 2010.

Rank, O. 1909. Le mythe de la naissance du héros, Paris, Payot, 1983.

Tasca, N. ; Seabra, J.A. 2002. « Mythe et poésie ", dans C. Bottela (sous la direction de), Penser les limites, Paris, Delachaux et Niestlé, p. 167-175.

TURKLE, S. 2012. Alone together : why we expect more from technology and less from each orher, New York, Basic Books.

Valleur, M. ; Matysiak, J.-C. 2002. Les addictions, Panorama clinique, modèles explicatifs, débat social et prise en charge, Paris, Armand Colin.

\section{The virtual hero as revealer of archaic phantasms at adolescence}

KEYWORDS

Adolescence, phantasy, hero, virtual.

\section{Abstract}

The figure of the hero is no longer limited to that of the mythical hero ; the virtual hero henceforth provides a contemporary transformation to occupy this position in the phantasy world of adolescents. But do these two heroes cover the same internal issues? Through analysis of a case related to clinical research, we propose to explore investment in the heroic avatar through three approaches that are at the centre of the process of adolescence : instinctual turmoil, Narcissistic and objectal conflictuality, and the problem of loss. What emerges from comparison by analogy between the libidinal investment of ancient heroes and virtual heroes is the divergence that develops in this clinical situation between imagining being a hero and feeding virtually the megalomaniac phantasy of being a hero. 